\title{
RENTABILIDAD DE BATERİAS EN APLICACIONES FV PARA EL SECTOR COMERCIAL BAJO OPERACIÓN BASADA EN MPC
}

\author{
P. Ayuso, E. Pérez, J. Cardo-Miota y H. Beltran \\ Departamento de Ingeniería de Sistemas Industriales y Diseño, Universitat Jaume I, \\ Castelló de la Plana, España pereze@uji.es // ORCID: 0000-0001-8466-3941
}

\begin{abstract}
Resumen
Este trabajo define una estrategia de operación basada en Control Predictivo basado en Modelo (Model Predictive Control, $M P C$ ) para instalaciones fotovoltaicas con baterías instaladas en el sector terciario/comercial. La propuesta incluye modelos de predicción de la irradiancia a futuro y modelos de consumo de las cargas basados en técnicas de agrupamiento. La operación del sistema se simula durante un año entero con datos de irradiancia $y$ consumo reales para un centro comercial situado al sur de España. Finalmente, se analiza la rentabilidad del sistema, en términos de vida útil de las baterías requeridas para lograr el retorno de la inversión realizada, para diferentes combinaciones de sistema FV, tamaño de las baterías, y precios de la energía.
\end{abstract}

Palabras clave: aplicaciones fotovoltaicas, control predictivo basado en modelo, mercados eléctricos, sistemas de almacenamiento de energía.

\section{INTRODUCCIÓN}

Con el continuo aumento de su cuota de mercado durante los últimos años, la fotovoltaica (FV) es hoy en día la tecnología renovable líder en MW instalados al año a nivel mundial después de superar a la energía eólica en 2016 [6]. En total, la capacidad FV global instalada a finales de 2020 es superior a $600 \mathrm{GW}$, según las estimaciones [19].

Sin embargo, las aplicaciones FV presentan varios desafíos principalmente asociados al carácter estocástico de su producción [9], aunque se han hecho varios esfuerzos en esta dirección [17]. Debido a esto y gracias a una disminución progresiva de los precios [14] de los sistemas de almacenamiento de energía, especialmente en el caso de las baterías de iones de litio (BIL), estas están comenzando a convertirse en un elemento importante en las instalaciones FV. De hecho, la combinación de FV y BIL es una solución disponible comercialmente para aplicaciones residenciales [5] y hay muchas propuestas en la literatura que se centran en varios aspectos de su funcionamiento abarcando desde el tamaño óptimo del sistema de baterías [18] hasta la maximización del beneficio económico obtenido con su introducción [15].

Por otro lado, el uso de BIL como reserva energética para mejorar la producción de plantas FV también ha sido una línea de investigación importante. Esta mejora se puede obtener de varias formas, dependiendo de los objetivos de la propuesta: suavizado de potencia [11], control de rampa [2], control de tensión [8], entre otros.

Sin embargo, las BIL junto con plantas fotovoltaicas de tamaño intermedio, como las que podrían implementarse para un centro comercial, han recibido mucha menos atención. La falta de interés de este tipo de instalación en el sector comercial puede estar relacionada con el hecho de que las baterías siguen siendo un elemento costoso que aumenta la inversión requerida. Además, como el beneficio económico de utilizar una BIL, así como su esperanza de vida útil, es difícil de evaluar, la rentabilidad de dicha inversión puede quedar en duda.

Existen varios modelos para predecir la expectativa de vida útil de las BIL [1], [3], con diferentes enfoques entre ellas y dedicadas a diferentes químicas. Sin embargo, la mayoría de ellos están de acuerdo en que existen mecanismos de envejecimiento relacionados con el paso del tiempo (envejecimiento por calendario) y otros mecanismos relacionados con la operación (envejecimiento por ciclado). En particular, el número y la naturaleza de los ciclos (profundidad de descarga y estado de carga promedio) que experimenta la BIL, junto con la temperatura, son los factores principales para el envejecimiento por ciclado.

Este trabajo tiene como objetivo proporcionar una estimación detallada de la potencial rentabilidad económica de las instalaciones FV con BIL aplicadas en el sector comercial. Para ello, se focaliza el esfuerzo en analizar cuál debería ser la vida útil mínima de las baterías para que su instalación sea rentable, partiendo de una curva de demanda y de irradiancia conocidas. Esta información, junto con la expectativa de vida útil de la batería, debería ser suficiente para tomar las decisiones de 
inversión adecuadas.

Con todo, el documento se organiza como sigue: en la sección 2 se define una estrategia de operación del sistema para determinar los ahorros brutos anuales con diferentes tamaños de instalación, tanto de la FV como de las BIL. En la sección 3 se define los modelos para la predicción de irradiancia y de perfil de carga. En la sección 4 se presentan los resultados tanto de operación del sistema como de rentabilidad de instalaciones con diferentes tamaños. Finalmente, en la sección 5 se obtienen algunas conclusiones.

\section{OPERACIÓN DEL SISTEMA BAJO MPC}

El estudio propuesto en este trabajo requiere definir de partida cómo va a operar el sistema FV con BIL.

En este sentido, hay que tener en cuenta que, para cada instante, hay dos variables incontrolables en la operación: la producción de los paneles fotovoltaicos $\left(P_{F V}\right)$ y el consumo de las cargas $\left(P_{C}\right)$. En general, estas potencias no serán iguales y, cuando $P_{C}>P_{F V}$, la producción de paneles deberá complementarse ya sea desde la descarga de las BIL $\left(P_{B I L}\right)$ o de la red eléctrica $\left(P_{r e d}\right)$. Por el contrario, cuando $P_{C}<P_{F V}$, la energía excedente puede enviarse a la BIL o venderse a red. $P_{B I L}$ y $P_{\text {red }}$ son, por lo tanto, variables controlables y hay que decidir si es mejor que el sistema use una $u$ otra en cada situación.

En una estructura de precios constantes durante todo el día, en la que los precios de compra de la red son siempre más altos que los de venta pero sin cambiar ninguno de ellos a lo largo de la jornada, la estrategia óptima siempre sería cargar o descargar la BIL antes que intercambiar energía con la red.

Sin embargo, los precios cambian en diferentes períodos del día en la mayoría de los mercados de electricidad, siendo más altos en períodos de alta demanda (horas pico) y más bajos en los de baja demanda (horas valle). En este contexto, la estrategia óptima podría ser diferente de la descrita anteriormente ya que se puede plantear el caso de que sea conveniente ahorrar energía en la BIL durante las horas valle si esta se va a demandar durante los períodos pico.

Este tipo de problema, que requiere tener en cuenta el efecto que tienen las acciones actuales en la optimización y en la capacidad de cumplir con las restricciones en el futuro es muy adecuado para la aplicación del MPC.

MPC [4] es una técnica de diseño de controlado- res basada en una estrategia de optimización en la que los resultados futuros para un horizonte $\mathrm{N}$ dado, llamado horizonte de predicción, se predicen en cada instante utilizando modelos de proceso. Estas salidas predichas dependen de las señales de control futuras, que se calculan optimizando un criterio determinado y cumpliendo un conjunto de restricciones. Aunque se calcula una secuencia completa de señales de control futuras, solo la primera se envía efectivamente al proceso, porque en el siguiente periodo de muestreo estará disponible nueva información. Esto se conoce como horizonte móvil.

Para el sistema FV con baterías, el criterio de optimización está determinado principalmente por el equilibrio económico de la energía intercambiada con la red. Sin embargo, como se discutirá más adelante, también es conveniente incluir la potencia inyectada o extraída de la BIL. En cuanto a las restricciones, debe cumplirse el equilibrio de potencia, incluidas las potencias del sistema $\mathrm{FV}$, la BIL, la red y las cargas; también el estado de carga (SOC, por sus siglas en inglés) de la BIL debe mantenerse dentro de sus límites, así como la potencia intercambiada con la red y por la BIL que también están límitadas.

Debe tenerse en cuenta que la producción de los paneles FV y el consumo de las cargas a futuro deben conocerse de alguna manera para garantizar el cumplimiento del equilibrio de potencia. Sin embargo, estas variables son imposibles de conocer con antelación, ya que dependen principalmente de la irradiación futura del sol y del comportamiento de los consumidores, respectivamente. Por lo tanto, se emplearán los modelos de predicción correspondientes $\left(\begin{array}{lll}\hat{P}_{F V} & \text { y } & \hat{P}_{C}\end{array}\right)$ descritos en la siguiente sección.

Con todas estas consideraciones, y siguiendo un enfoque similar a [12], el problema de optimización a resolver bajo un MPC se puede formular de la siguiente manera:

$$
\begin{array}{rl}
\min J_{N}=\sum_{k=0}^{N} & T\left(c_{r e d}(t+k) P_{r e d}(t+k)\right. \\
& \left.+c_{B I L}(t+k)\left|P_{B I L}(t+k)\right|\right)
\end{array}
$$

Sujeto para $k=0 \ldots N$ a:

$$
\begin{gathered}
\hat{P}_{F V}(t+k)+P_{r e d}(t+k)+P_{B I L}(t+k)=\hat{P}_{C}(t+k) \\
E_{B I L}(t+k+1)=E_{B I L}(t+k)-T \cdot \eta \cdot P_{B I L}(t+k) \\
E_{B I L, \text { min }} \leq E_{B I L}(t+k) \leq E_{B I L, \text { max }} \\
P_{B I L, \text { min }} \leq P_{B I L}(t+k) \leq P_{B I L, \text { max }} \\
P_{\text {red,min }} \leq P_{\text {red }}(t+k) \leq P_{\text {red,max }}
\end{gathered}
$$

donde: 
- $T$ es el periodo de muestreo.

- $\hat{P}_{F V}(t+k)$ y $\hat{P}_{C}(t+k)$ son las predicciones para $P_{F V}(t+k)$ y $P_{C}(t+k)$, respectivamente.

- $P_{\text {red }}(t+k)$ es la potencia intercambiada con la red en el intante $t+k$, con $P_{\text {red }}(t+k)>0$ cuando se compra energía.

- $P_{B I L}(t+k)$ es la potencia intercambiada con las BIL en el intante $t+k$, con $P_{B I L}(t+k)>0$ cuando se descarga.

- $E_{B I L}(t+k)$ es la energía disponible en las BIL en el intante $t+k$.

- $P_{B I L, \min }, P_{B I L, \max }, P_{\text {red,min }}$ y $P_{\text {red,max }}$ son los límites inferior y superior para la potencia intercambiada con la BIL y la red. Estas restricciones se deben a las limitaciones de los convertidores de potencia y, por lo tanto: $P_{B I L, \min }=-P_{B I L, \max }$ y $P_{r e d, \min }=$ $-P_{\text {red,max }}$

- $E_{B I L, \min }$ y $E_{B I L, \max }$ son los límites entre los cuales se debe mantener el SOC de las BIL.

- $c_{B I L}(t+k)$ es el coste de intercambiar energía con las BIL, usado para ajustar el comportamiento del sistema.

- $c_{r e d}=\left\{\begin{array}{ll}C_{\text {compra }}(h) & \text { para } P_{\text {red }}>0 \\ C_{\text {venta }}(h) & \text { para } P_{\text {red }}<0\end{array}\right.$ son los precios de electricidad, los cuales cambian de valor en función de la hora del día $h$ y de la tarifa, con $c_{\text {compra }}(h)>c_{\text {venta }}(h)$ para cualquier $h$.

- $\eta=\left\{\begin{array}{ll}\frac{1}{\eta_{c r g}} & \text { para } P_{B I L}>0 \\ \eta_{\text {dcrg }} & \text { para } P_{B I L}<0\end{array}\right.$, siendo $\eta_{d c r g} \mathrm{y}$ $\eta_{\text {crg }}$ las eficiencias de descarga y carga, respectivamente.

Aunque la mayoría de las ecuaciones del problema anterior son lineales, sigue siendo un problema complejo de resolver, ya que presenta dos funciones por partes, $c_{\text {red }}$ y $\eta$, y una función no lineal, $\left|P_{B I L}(t+k)\right|$. Este tipo de funciones pueden tratarse mediante la introducción de variables binarias, que conducen a una optimización lineal entera mixta (MILP, por sus siglas en inglés), computacionalmente compleja para el tamaño del problema. Por lo tanto, se propone una formulación diferente.

La idea es reemplazar $P_{\text {red }}$ por dos nuevas variables, $P_{\text {compra }}$ y $P_{\text {venta }}$, para los casos en que es positivo o negativo. Del mismo modo, $P_{B I L}$ es reemplazada por $P_{c r g}$ y $P_{d c r g}$, y el coste de la energía intercambiada por las BIL, $c_{B I L}$, también es sustituido por $c_{c r g}$ y $c_{d c r g}$.
Además, con la formulación anterior, la optimización podría presentar múltiples soluciones con el mismo mínimo, debido a la naturaleza constante de los costes por periodos horarios. Para evitar esto, se introducen dos secuencias de ponderación, $\lambda_{c}(k)=\alpha^{k}$ y $\lambda_{d}(k)=\beta^{k} . \lambda_{c}(k)$, con $\alpha=0,999$, pondera a $P_{c r g}$ de manera que, en situaciones iguales, favorezca la carga lo más tarde posible. Por el contrario, $\lambda_{d}(k)$, con $\beta=1,001$, pondera a $P_{d c r g}$ para favorecer la descarga lo antes posible.

Con los cambios introducidos, el problema de optimización pasa a ser:

$$
\begin{aligned}
\min _{N} & =\sum_{k=0}^{N} T\left(c_{\text {compra }}(t+k) P_{\text {compra }}(t+k)\right. \\
& -c_{\text {venta }}(t+k) \cdot P_{\text {venta }}(t+k) \\
& +\lambda_{c}(k) \cdot c_{\text {crg }}(t+k) \cdot P_{\text {crg }}(t+k) \\
& \left.+\lambda_{d}(k) \cdot c_{d c r g}(t+k) \cdot P_{d c r g}(t+k)\right)
\end{aligned}
$$

Sujeto para $k=0 \ldots N$ a:

$$
\begin{gathered}
\hat{P}_{F V}(t+k)+P_{\text {compra }}(t+k)+P_{d c r g}(t+k) \\
=\hat{P}_{C}(t+k)+P_{\text {venta }}(t+k)+P_{\text {crg }}(t+k) \\
E_{B I L}(t+k+1)=E_{B I L}(t+k) \\
+T\left(\eta_{c r g} \cdot P_{c r g}(t+k)-\frac{1}{\eta_{d c r g}} \cdot P_{d c r g}(t+k)\right) \\
E_{B I L, \text { min }} \leq E_{B I L}(t+k) \leq E_{B I L, \text { máx }} \\
0 \leq P_{\text {compra }}(t+k) \leq P_{\text {red,máx }} \\
0 \leq P_{\text {venta }}(t+k) \leq-P_{\text {red,máx }} \\
0 \leq P_{\text {crg }}(t+k) \leq P_{B I L, \text { máx }} \\
0 \leq P_{d c r g}(t+k) \leq-P_{B I L, \text { minn }} \\
P_{\text {compra }}(t+k) \cdot P_{\text {venta }}(t+k)=0 \\
P_{\text {crg }}(t+k) \cdot P_{d c r g}(t+k)=0
\end{gathered}
$$

Debe tenerse en cuenta que, con esta nueva formulación, la función objetivo y todas las restricciones, excepto las dos últimas, son lineales. Estas dos restricciones finales de igualdad cuadrática se introducen para evitar soluciones ilógicas del problema de optimización, como evitar que se produzca al mismo tiempo la compra y venta de energía con la red, o la carga y descarga de las BIL.

Sin embargo, a partir de la definición de la función objetivo en sí, se puede deducir que nunca habría una solución óptima del problema con $P_{\text {compra }} \neq 0$ y $P_{\text {venta }} \neq 0$, incluso sin incluir la restricción cuadrática. De hecho, como $c_{\text {compra }}>c_{\text {venta }}$, cualquier solución con $P_{\text {compra }}>P_{\text {venta }}>0$ es subóptimo con respecto a $P_{\text {compra }}^{\prime}=P_{\text {compra }}-P_{\text {venta }} \mathrm{y}$ $P_{\text {venta }}^{\prime}=0$. Por lo tanto, la restricción cuadrática es redundante y puede descartarse. Para obtener 
este mismo comportamiento con $P_{c r g}$ and $P_{d c r g}$, se introducen costes distintos para la carga y la descargar, ambos cercanos a 0 .

Atendiendo a todas estas consideraciones, el problema de optimización se puede definir como una optimización lineal (LP, por sus siglas en inglés), que se puede resolver fácilmente con herramientas de optimización estándar.

Las secuencias de potencia obtenidas mediante LP son óptimas solo si la generación FV y el consumo real son iguales a los modelos de predicción $\hat{P}_{F V}$ y $\hat{P}_{C}$, respectivamente. Sin embargo, en general este no será el caso y, por lo tanto, las secuencias pueden ser incluso no factibles. En un MPC, este hecho es especialmente crítico para los primeros valores de dicha secuencia, que son los que realmente se aplican. Para los casos en que esto ocurre, (normalmente porque no se alcanza el equilibrio de potencia), se decide mantener el valor de $P_{B I L}(t)$ obtenido en la optimización y variar $P_{\text {red }}(t)$ para que dicho balance se cumpla.

\section{CLUSTERING DE IRRADIANCIA Y PERFIL DE CARGA}

Los modelos de predicción propuestos en este trabajo están basados en un análisis de agrupamiento o "clustering", que es una forma de agrupar los datos (en nuestro caso los perfiles horarios de $P_{F V}$ o $P_{C}$ ) de forma que los perfiles clasificados en un mismo clúster sean más similares entre sí (según un criterio específico) que a los de otros clústers. Por consiguiente, a los días clasificados dentro de cada clúster se les asigna como modelo de predicción el perfil del centroide correspondiente a su clúster.

Además, existen diferentes métodos diseñados para ayudar a encontrar el número apropiado de clústers. En este artículo se aplican los métodos más utilizados en la literatura, el método del codo [10] y el estadístico Gap [16]. El método del codo está basado en el algoritmo $k$-means, que divide los datos en $k$ clústers distintos en función de la distancia euclidiana al cuadrado de los datos clasificados al centroide del clúster. Así, este método se basa en calcular, para diferentes valores de $k$, la suma de todas las distancias de cada grupo de datos al centroide de su clúster. Finalmente, determina gráficamente cuándo el decremento de esta suma, que desciende continuamente con $k$, no es lo suficientemente significativo para seguir aumentando el número de clústers. Por otro lado, el estadístico Gap usa la salida del algoritmo de clustering para comparar el cambio en la dispersión dentro del clúster con el esperado bajo una distribución apropiada de referencia nula. Para la aplicación considerada en esta propuesta, se utilizan datos reales de consumo durante todo un año de un centro comercial en España. La Figura 1 representa el número óptimo de clústers para el consumo anual utilizando ambos métodos de clustering que coinciden en un número óptimo de 3 clústers.

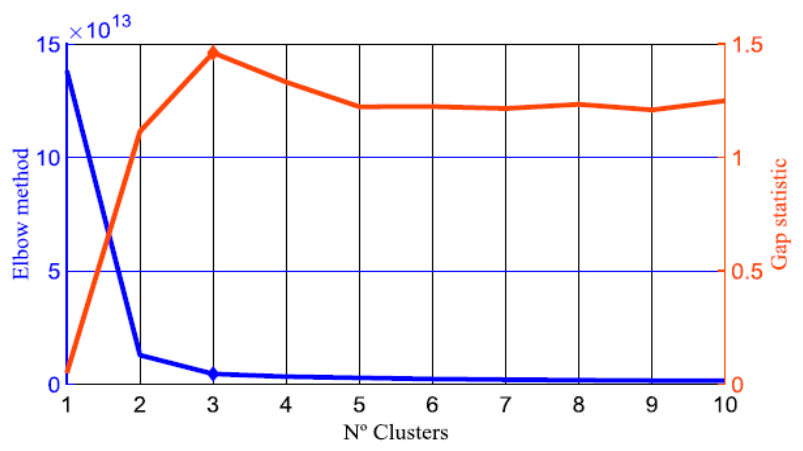

Figura 1: Número óptimo de clústers para las curvas de consumo diario de un centro comercial

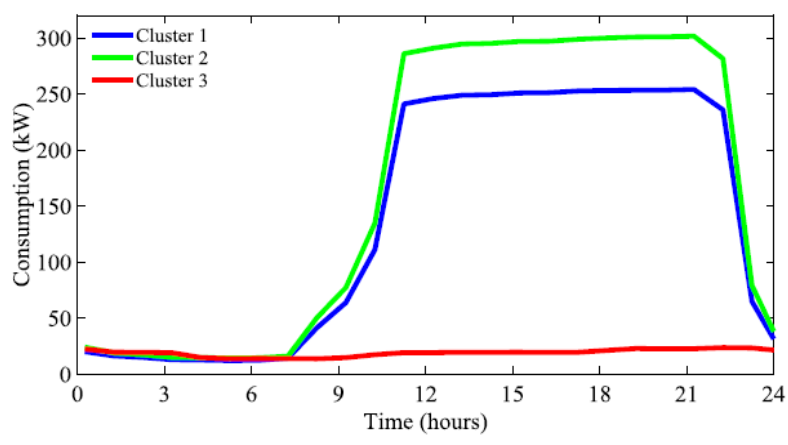

Figura 2: Clustering anual de las curvas de consumo diario de un centro comercial

La Figura 2 muestra los tres perfiles de carga del consumo anual que se utilizarán como $\hat{P}_{C}$. Esto parece un resultado lógico ya que los clústers 1 y 2 podrían representar el consumo correspondiente a días laborales de invierno y verano [13], respectivamente, mientras el clúster 3 agrupa los días festivos, cuando el centro comercial está cerrado al público.

En cuanto a la producción FV, se utilizan los datos reales de irradiancia de todo un año en una ciudad del sur de España. Al igual que se ha presentado para agrupar las curvas del consumo anual de un centro comercial, el número óptimo de clústers para la irradiancia anual también puede ser obtenido utilizando el método del codo y el estadístico Gap, que muestran que el número óptimo de clústers es 5.

Los centroides de estos clústers, mostrados en la Figura 3 , se utilizarán como $\hat{P}_{F V}$ y ofrecen re- 
sultados explicables. Se puede observar cómo los clústers 3 y 4 representan la irradiancia correspondiente a los días soleados de invierno y verano, respectivamente. De hecho, el clúster 3 muestra un pico más bajo y un perfil en el que la irradiancia aparece más tarde y termina antes que el clúster 4 , que es un comportamiento esperado de los días de invierno con respecto a los de verano. Además, el clúster 5 muestra el perfil de irradiancia de los días soleados por la mañana, pero nublados por la tarde. Por el contrario, el clúster 2 muestra el perfil de irradiancia de los días nublados por la mañana, pero soleados por la tarde. Por último, el clúster 1 muestra la irradiancia en los días nublados.

La Figura 4 muestra, a modo de ilustración, el centroide y todas las curvas de irradiancia del clúster 5. De esta forma, para la simulación en próximas secciones, $P_{P V}$ será una curva como las que aparecen en color en la Figura 4 , mientras que $\hat{P}_{F V}$ será el centroide del clúster (en negro).

\section{RESULTADOS Y DISCUSIÓN}

Una vez presentado el algoritmo propuesto para la operación de la planta, y los modelos de predicción que este necesita, se analiza la rentabilidad de la BIL, en términos del tiempo necesario para recuperar la inversión. Como se ha introducido anteriormente, este tiempo debe ser inferior a la vida útil prevista para las BIL.

Para realizar este análisis, es necesario realizar algunas definiciones del sistema. En este sentido, se consideran tres alternativas diferentes de potencia nominal de la planta FV: $350 \mathrm{~kW}, 250 \mathrm{~kW}$ y 150 kW que están, respectivamente, un poco por encima, un poco por debajo y muy por debajo del nivel de carga máxima del centro comercial. En cuanto a la potencia máxima de la BIL, se fija en el $80 \%$ de la nominal de la planta FV. Finalmente, se consideran dos valores de capacidad de almacenamiento de la BIL para cada uno de los casos

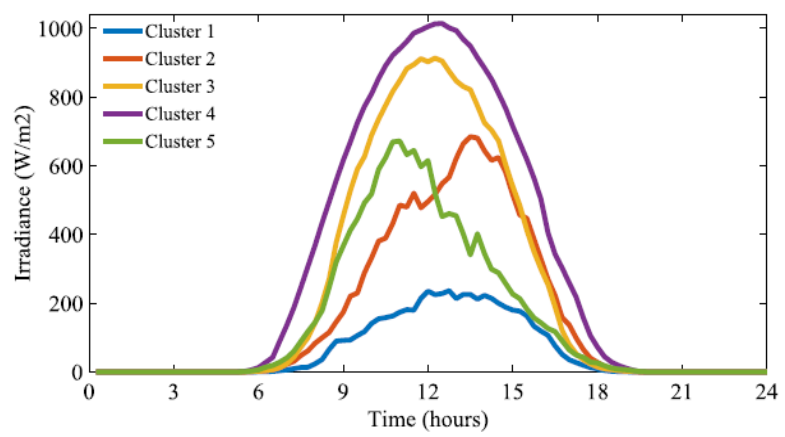

Figura 3: Centroides del clustering de la irradiancia anual anteriores: 1 o 2 horas de acumulación a plena potencia. A modo de comparación, cabe destacar que también se incluye en el análisis el caso sin BIL.

Por último, también es necesario definir los precios de la electricidad de la red. Se analizarán 2 estructuras diferentes de precios, mostradas en la Tabla 1.

La Figura 5 muestra cómo gestiona la planta el algoritmo MPC propuesto durante dos días consecutivos para uno de los casos considerados: el sistema FV de $350 \mathrm{~kW}$ con una BIL de $560 \mathrm{kWh}$ bajo los precios de la tarifa 2. Las entradas del algoritmo MPC, es decir, las predicciones de la potencia consumida por el centro comercial y la potencia generada por la instalación FV son mostrados en negro y azul, respectivamente. Las salidas del algoritmo, es decir, la energía intercambiada con la red y con la BIL, se muestran en rojo y verde, respectivamente. Cabe destacar que un valor de $P_{\text {red }}$ positivo representa compra, mientras que un $P_{B I L}$ positivo representa una descarga de las BIL. En la Figura 6 se muestra la evolución del SOC de las BIL y los precios de compra de la electricidad.

A partir del análisis de ambas figuras, se puede observar como el optimizador decide si cargar la BIL en el periodo valle en función de la irradiancia esperada para el siguiente día. Así, en el primer día mostrado en las Figuras 5 y 6 , la BIL se carga durante las horas valle hasta el $75 \%$ del SOC, dado que durante el resto del día la generación $\mathrm{FV}$ superará el consumo permitiendo cargar la batería hasta el valor máximo del SOC. Sin embargo, en el caso del segundo día, la generación FV no supera el consumo, por lo que se decide cargar completamente la batería en el periodo de menor coste. Además, la introducción de $\lambda_{c}(k)$ y $\lambda_{d}(k)$ obliga a la BIL a descargarse lo antes posible mientras está en periodo punta y a cargar en el periodo valle lo más tarde posible. Por lo tanto, la batería se mantiene al mínimo SOC el mayor tiempo posible para reducir su envejecimiento por calendario.

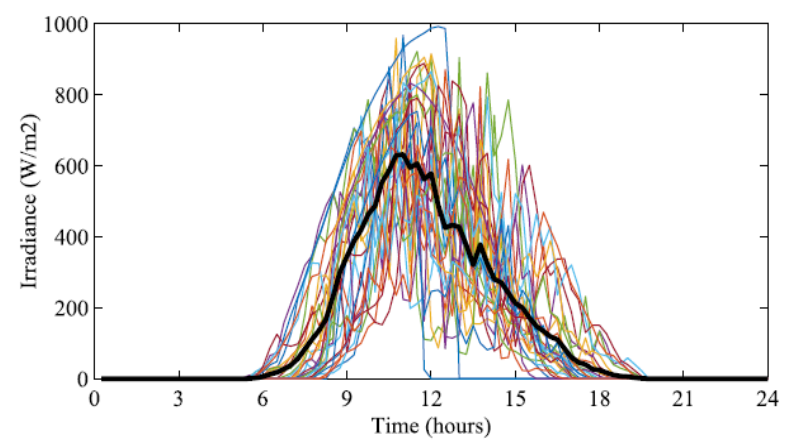

Figura 4: Ejemplo de clustering de un grupo de perfiles de irradiancia 


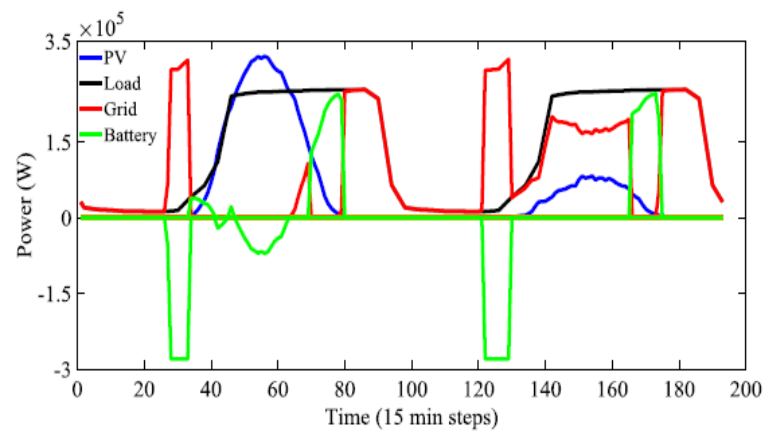

Figura 5: Resultados iniciales del optimizador

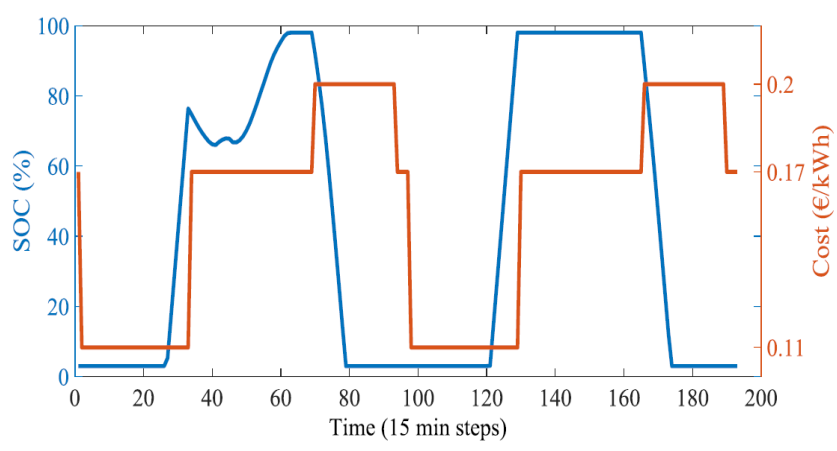

Figura 6: Evolución de SOC y $c_{\text {compra }}$

Como se ha introducido en la sección 2, en general las predicciones para $\hat{P}_{F V}$ y $\hat{P}_{C}$ no coinciden con los valores reales de $P_{F V}$ y $P_{C}$ que experimenta el sistema, por lo que se decide mantener siempre el $P_{B I L}$ dado por la optimización y ajustar $P_{\text {red }}$ para garantizar el cumplimiento del equilibrio de potencias. La Figura 7 muestra la evolución de potencias en el caso real, con $P_{\text {red }}$ ajustado. Obsérvese que, como $P_{B I L}$ no cambia, la evolución del SOC en este caso es la misma que en la Figura 6. La diferencia principal entre la Figura 5 y la Figura 7 se observa en el segundo día, cuando, debido a los errores en $\hat{P}_{F V}$, es necesario comprar más energía a la red. También se observa una pequeña diferencia que lleva a un comportamiento ligeramente subóptimo durante el primer día, en el que los errores de predicción hacen que el sistema venda algo de energía a la red, ya que hay cierto exceso de energía porque el consumo de la carga es un poco inferior al previsto.

Para el análisis de rentabilidad, se realizan simulaciones del sistema para todo un año en las condiciones descritas anteriormente. Para cada caso, se calcula el coste de la compra de energía, con y sin

Tabla 1: Costes de la energía

\begin{tabular}{|c|c|c|c|c|c|c|}
\cline { 2 - 7 } \multicolumn{1}{c|}{} & \multicolumn{3}{c|}{ Compra $(€ / \mathrm{kWh})$} & \multicolumn{3}{c|}{ Venta $(€ / \mathrm{kWh})$} \\
\cline { 2 - 7 } \multicolumn{1}{c|}{} & Valle & Estándar & Pico & Valle & Estándar & Pico \\
\hline Tarifa 1 & 0,122 & 0,150 & 0,160 & 0,038 & 0,047 & 0,050 \\
\hline Tarifa 2 & 0,110 & 0,170 & 0,200 & 0,038 & 0,047 & 0,050 \\
\hline
\end{tabular}

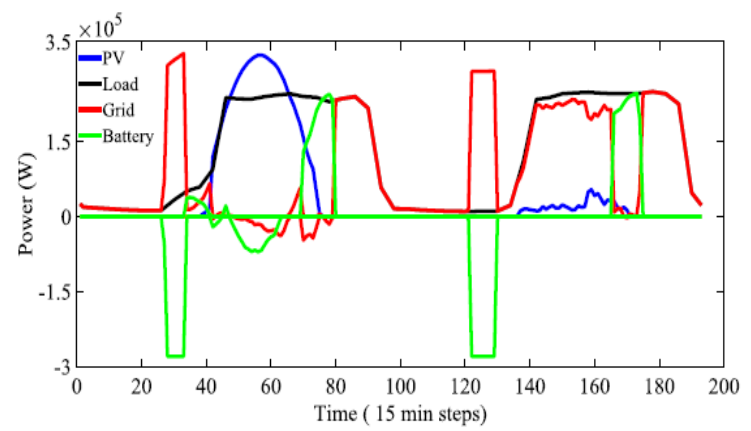

Figura 7: Evolución de potencias ajustadas con Pred

el sistema híbrido FV con BIL. Además, cuando se considera el sistema híbrido, también se calculan los ingresos obtenidos por la venta de la energía excedente y los costes operativos, definidos como el coste de las compras menos los ingresos. La diferencia entre el coste de las compras sin el sistema híbrido y estos costes operativos representa el ahorro bruto anual conseguido con la introducción de dicho sistema. En las tablas 2 y 3 se muestran los resultados para los distintos casos considerados.

Además, estas tablas introducen los costes de las baterías, considerando un ratio de $187 € / \mathrm{kWh}[7]$ y la vida útil mínima para la rentabilidad. Obsérvese que, incluso sin baterías, la aplicación $\mathrm{FV}$ por sí misma produce ahorros. Por tanto, la vida útil mínima se calcula como:

$$
\text { Mín.vidaútil }=\frac{\text { Coste de batería }}{\text { Ahorros }- \text { Ahorros }_{0 k W h}}
$$

A partir de los resultados obtenidos y considerando las vidas útiles típicas de las BIL, se puede concluir que en con los precios de la tarifa 1 es difícil asegurar la rentabilidad de la BIL en ninguno de los casos considerados. Sin embargo, con los de la segunda tarifa, algunas combinaciones permiten recuperar la inversión en un adecuado número de años (hasta 7). Además, también se concluye que cuanto más alto sea el valor de la potencia nominal del sistema FV, más fácil será conseguir un retorno de la inversión positivo.

También es interesante comprobar que, para una potencia fija de la BIL, el aumento de la capacidad reduce tanto el coste de las compras como los ingresos por las ventas. Esto se debe a que, cuando se dispone de más almacenamiento, es más eficaz ahorrar energía y evitar futuras compras para venderlas a un precio mucho menor. Sin embargo, en la mayoría de los casos, este incremento de capacidad también implica un aumento del coste de la batería, y, en consecuencia, alarga la vida útil mínima para la rentabilidad. 
Tabla 2: Resultados de la optimización de la tarifa 1

\begin{tabular}{|c|c|c|c|c|c|c|c|c|c|}
\hline \multirow{2}{*}{$\begin{array}{l}\text { Potencia } \\
\text { FV }\end{array}$} & \multirow{2}{*}{$\begin{array}{l}\text { Potencia } \\
\text { de BIL }\end{array}$} & \multirow{2}{*}{$\begin{array}{l}\text { Capacidad } \\
\text { de BIL }\end{array}$} & \multicolumn{2}{|c|}{ Coste compras } & \multirow{2}{*}{$\begin{array}{c}\text { Ingresos venta } \\
\text { de energía } \\
\text { con FV+BIL }\end{array}$} & \multirow{2}{*}{$\begin{array}{l}\text { Costes operativos } \\
\text { con FV+BIL }\end{array}$} & \multirow{2}{*}{$\begin{array}{c}\text { Ahorro } \\
\text { bruto anual }\end{array}$} & \multirow{2}{*}{$\begin{array}{c}\text { Coste } \\
\text { de BIL }\end{array}$} & \multirow{2}{*}{$\begin{array}{c}\text { Vida útil } \\
\text { mínima para } \\
\text { la rentabilidad }\end{array}$} \\
\hline & & & $\sin \mathbf{F V}+\mathbf{B I L}$ & $\operatorname{con} \mathbf{F V}+\mathrm{BIL}$ & & & & & \\
\hline \multirow{3}{*}{$150 \mathrm{~kW}$} & \multirow{3}{*}{$120 \mathrm{~kW}$} & $0 \mathrm{kWh}$ & $168 \mathrm{k} €$ & $132 \mathrm{k} €$ & $1 \mathrm{k} €$ & $131 \mathrm{k} €$ & $37 \mathrm{k} €$ & - & - \\
\hline & & $120 \mathrm{kWh}$ & $168 \mathrm{k} €$ & $131 \mathrm{k} €$ & $1 \mathrm{k} €$ & $130 \mathrm{k} €$ & $38 \mathrm{k} €$ & $22 \mathrm{k} €$ & 22 \\
\hline & & $240 \mathrm{kWh}$ & $168 \mathrm{k} €$ & $130 \mathrm{k} €$ & $1 \mathrm{k} €$ & $129 \mathrm{k} €$ & $39 \mathrm{k} €$ & $45 \mathrm{k} €$ & 22 \\
\hline \multirow{3}{*}{$250 \mathrm{~kW}$} & \multirow{3}{*}{$200 \mathrm{~kW}$} & $0 \mathrm{kWh}$ & $168 \mathrm{k} €$ & $110 \mathrm{k} €$ & $3 \mathrm{k} €$ & $107 \mathrm{k} €$ & $61 \mathrm{k} €$ & - & - \\
\hline & & $200 \mathrm{kWh}$ & $168 \mathrm{k} €$ & $108 \mathrm{k} €$ & $2 \mathrm{k} €$ & $106 \mathrm{k} €$ & $62 \mathrm{k} €$ & $37 \mathrm{k} €$ & 24 \\
\hline & & $400 \mathrm{kWh}$ & $168 \mathrm{k} €$ & $106 \mathrm{k} €$ & $2 \mathrm{k} €$ & $104 \mathrm{k} €$ & $64 \mathrm{k} €$ & $75 \mathrm{k} €$ & 24 \\
\hline \multirow{3}{*}{$350 \mathrm{~kW}$} & \multirow{3}{*}{$280 \mathrm{~kW}$} & $0 \mathrm{kWh}$ & $168 \mathrm{k} €$ & $95 \mathrm{k} €$ & $6 \mathrm{k} €$ & $89 \mathrm{k} €$ & $79 \mathrm{k} €$ & - & - \\
\hline & & $280 \mathrm{kWh}$ & $168 \mathrm{k} €$ & $89 \mathrm{k} €$ & $4 \mathrm{k} €$ & $84 \mathrm{k} €$ & $84 \mathrm{k} €$ & $52 \mathrm{k} €$ & 11 \\
\hline & & $560 \mathrm{kWh}$ & $168 \mathrm{k} €$ & $86 \mathrm{k} €$ & $4 \mathrm{k} €$ & $82 \mathrm{k} €$ & $86 \mathrm{k} €$ & $105 \mathrm{k} €$ & 15 \\
\hline
\end{tabular}

Tabla 3: Resultados de la optimización de la tarifa 2

\begin{tabular}{|c|c|c|c|c|c|c|c|c|c|}
\hline \multirow{2}{*}{$\begin{array}{l}\text { Potencia } \\
\text { FV }\end{array}$} & \multirow{2}{*}{$\begin{array}{l}\text { Potencia } \\
\text { de BIL }\end{array}$} & \multirow{2}{*}{$\begin{array}{l}\text { Capacidad } \\
\text { de BIL }\end{array}$} & \multicolumn{2}{|c|}{ Coste compras } & \multirow{2}{*}{$\begin{array}{c}\text { Ingresos venta } \\
\text { de energía } \\
\text { con FV+BIL }\end{array}$} & \multirow{2}{*}{$\begin{array}{l}\text { Costes operativos } \\
\text { con FV+BIL }\end{array}$} & \multirow{2}{*}{$\begin{array}{c}\text { Ahorro } \\
\text { bruto anual }\end{array}$} & \multirow{2}{*}{$\begin{array}{c}\text { Coste } \\
\text { de BIL }\end{array}$} & \multirow{2}{*}{$\begin{array}{c}\text { Vida útil } \\
\text { mínima para } \\
\text { la rentabilidad }\end{array}$} \\
\hline & & & $\sin \mathbf{F V}+\mathrm{BIL}$ & $\operatorname{con} \mathrm{FV}+\mathrm{BIL}$ & & & & & \\
\hline \multirow{3}{*}{$150 \mathrm{~kW}$} & \multirow{3}{*}{$120 \mathrm{~kW}$} & $0 \mathrm{kWh}$ & $180 \mathrm{k} €$ & $145 \mathrm{k} €$ & $1 \mathrm{k} €$ & $143 \mathrm{k} €$ & $37 \mathrm{k} €$ & - & - \\
\hline & & $120 \mathrm{kWh}$ & $180 \mathrm{k} €$ & $142 \mathrm{k} €$ & $1 \mathrm{k} €$ & $140.717 €$ & $40 \mathrm{k} €$ & $22 \mathrm{k} €$ & 9 \\
\hline & & $240 \mathrm{kWh}$ & $180 \mathrm{k} €$ & $140 \mathrm{k} €$ & $1 \mathrm{k} €$ & $138 \mathrm{k} €$ & $42 \mathrm{k} €$ & $45 €$ & 9 \\
\hline \multirow{3}{*}{$250 \mathrm{~kW}$} & \multirow{3}{*}{$200 \mathrm{~kW}$} & $0 \mathrm{kWh}$ & $180 \mathrm{k} €$ & $122 \mathrm{k} €$ & $3 \mathrm{k} €$ & $120 \mathrm{k} €$ & $61 \mathrm{k} €$ & - & - \\
\hline & & $200 \mathrm{kWh}$ & $180 \mathrm{k} €$ & $118 \mathrm{k} €$ & $2 \mathrm{k} €$ & $116 \mathrm{k} €$ & $65 \mathrm{k} €$ & $37 \mathrm{k} €$ & 10 \\
\hline & & $400 \mathrm{kWh}$ & $180 \mathrm{k} €$ & $114 \mathrm{k} €$ & $2 \mathrm{k} €$ & $112 \mathrm{k} €$ & $69 \mathrm{k} €$ & $75 \mathrm{k} €$ & 10 \\
\hline \multirow{3}{*}{$350 \mathrm{~kW}$} & \multirow{3}{*}{$280 \mathrm{~kW}$} & $0 \mathrm{kWh}$ & $180 \mathrm{k} €$ & $107 \mathrm{k} €$ & $6 \mathrm{k} €$ & $101 \mathrm{k} €$ & $80 \mathrm{k} €$ & - & - \\
\hline & & $280 \mathrm{kWh}$ & $180 \mathrm{k} €$ & $98 \mathrm{k} €$ & $5 \mathrm{k} €$ & $94 \mathrm{k} €$ & $87 \mathrm{k} €$ & $52 \mathrm{k} €$ & 7 \\
\hline & & $560 \mathrm{kWh}$ & $180 \mathrm{k} €$ & $92 \mathrm{k} €$ & $4 \mathrm{k} €$ & $88 \mathrm{k} €$ & $92 \mathrm{k} €$ & $105 \mathrm{k} €$ & 8 \\
\hline
\end{tabular}

Finalmente, se puede concluir en términos de rentabilidad que, para los casos considerados, sigue siendo más eficaz aumentar la potencia máxima $\mathrm{FV}$, cuando es posible, que introducir una BIL.

\section{CONCLUSIONES}

Este artículo ha introducido una estrategia de operación, basada en MPC, para conducir el funcionamiento óptimo desde el punto de vista económico, de un sistema FV con baterías instalado en un centro comercial. Además, se han propuesto modelos de predicción de la producción de los paneles FV y de los consumos de las cargas, basados en técnicas de clustering. Por último, se ha analizado la rentabilidad económica derivada de la introducción de una instalación FV con baterías simulando el sistema, durante todo un año, bajo diferentes condiciones en cuanto a tamaño de la FV, capacidad de la batería y tarifas eléctricas. Los resultados muestran que, para una tarifa en la que existen diferencias suficientemente significativas entre los costes en horas punta y valle, y si el tamaño del sistema FV es adecuado, se consigue un retorno de la inversión positivo en menos años que la vida útil esperable para las baterías de iones de litio.

\section{Agradecimientos}

Los autores agradecen el apoyo financiero de la Universitat Jaume I de Castelló (España) por sus programas de investigación para fomentar la investigación científica y el desarrollo tecnológico. Este Trabajo se desarrolló bajo dicho programa dentro del proyecto con código UJI-B2017-26 y dentro de la beca con código PREDOC/2020/35.

\section{English summary}

\section{PROFITABILITY OF BATTERIES IN PV APPLICATIONS FOR THE COMMERCIAL SECTOR UNDER MPC OPERATION}

\begin{abstract}
This work defines a strategy of operation based on Model Predictive Control (MPC) for a PV application with an energy storage system (ESS) in the commercial sector. Prediction models of future irradiance and loads consumption based on clustering techniques are also introduced. The operation is simulated for a whole year with real irradiance data in a southern spanish location and with real consumption values from a shopping center. Finally, profitability of the system, in terms of required lifetime of the ESS for the return of the investment, is analyzed for different scenarios of PV system, battery capacities and energy prices.
\end{abstract}

Keywords: PV applications, model predictive control, electricity markets, energy storage systems. 


\section{Referencias}

[1] A. Barré, B. Deguilhem, S. Grolleau, M. Gérard, F. Suard, and D. Riu, "A review on lithium-ion battery ageing mechanisms and estimations for automotive applications," Journal of Power Sources, vol. 241, pp. 680689, nov 2013.

[2] H. Beltran, I. Tomas Garcia, J. C. AlfonsoGil, and E. Perez, "Levelized Cost of Storage for Li-Ion Batteries Used in PV Power Plants for Ramp-Rate Control," IEEE Transactions on Energy Conversion, vol. 34, no. 1, pp. 554$561,2019$.

[3] M. Berecibar, I. Gandiaga, I. Villarreal, N. Omar, J. Van Mierlo, and P. Van den Bossche, "Critical review of state of health estimation methods of Li-ion batteries for real applications," Renewable and Sustainable Energy Reviews, vol. 56, pp. 572-587, apr 2016.

[4] E. Camacho and C. Bordons, Model Predictive Control, jan 2004, vol. 13.

[5] K. Darcovich, E. R. Henquin, B. Kenney, I. J. Davidson, N. Saldanha, and I. BeausoleilMorrison, "Higher-capacity lithium ion battery chemistries for improved residential energy storage with micro-cogeneration," $A p$ plied Energy, vol. 111, pp. 853-861, 2013.

[6] Janet L. Sawin, Jay Rutovitz, Freyr Sverrisson, Hannah E. Murdock, and Rana Adib, "Renewables 2018, Global Status Report," Tech. Rep., 2018.

[7] Jeff St. John, "Report: Levelized Cost of Energy for Lithium-Ion Batteries Is Plummeting," Greentech Media, 2019.

[8] M. N. Kabir, Y. Mishra, G. Ledwich, Z. Y. Dong, and K. P. Wong, "Coordinated Control of Grid-Connected Photovoltaic Reactive Power and Battery Energy Storage Systems to Improve the Voltage Profile of a Residential Distribution Feeder," IEEE Transactions on Industrial Informatics, vol. 10, no. 2, pp. 967-977, 2014.

[9] F. Katiraei and J. R. Agüero, "Solar PV Integration Challenges," IEEE Power and Energy Magazine, vol. 9, no. 3, pp. 62-71, 2011.

[10] T. Kodinariya and P. Makwana, "Review on Determining of Cluster in K-means Clustering," International Journal of Advance Research in Computer Science and Management Studies, vol. 1, pp. 90-95, jan 2013.
[11] X. Li, D. Hui, and X. Lai, "Battery Energy Storage Station (BESS)-Based Smoothing Control of Photovoltaic (PV) and Wind Power Generation Fluctuations," IEEE Transactions on Sustainable Energy, vol. 4, no. 2, pp. 464-473, 2013.

[12] E. Perez, H. Beltran, N. Aparicio, and P. Rodriguez, "Predictive Power Control for PV Plants With Energy Storage," IEEE Transactions on Sustainable Energy, vol. 4, no. 2, pp. 482-490, 2013.

[13] REE, "Atlas de la demanda Eléctrica Española," Tech. Rep., 1998.

[14] O. Schmidt, S. Melchior, A. Hawkes, and I. Staffell, "Projecting the Future Levelized Cost of Electricity Storage Technologies," Joule, vol. 3, no. 1, pp. 81-100, 2019.

[15] J. Segarra-Tamarit, E. Perez, J. C. AlfonsoGil, C. Arino, N. Aparicio, and H. Beltran, "Optimized management of a residential microgrid using a solar power estimation database," in 2017 IEEE 26th International Symposium on Industrial Electronics (ISIE), 2017, pp. 993-998.

[16] R. Tibshirani, G. Walther, and T. Hastie, "Estimating the Number of Clusters in a Data Set Via the Gap Statistic," Journal of the Royal Statistical Society Series B, vol. 63, pp. 411-423, feb 2001.

[17] C. Voyant, G. Notton, S. Kalogirou, M.-L. Nivet, C. Paoli, F. Motte, and A. Fouilloy, "Machine learning methods for solar radiation forecasting: A review," Renewable Energy, vol. 105, pp. 569-582, may 2017.

[18] J. Weniger, T. Tjaden, and V. Quaschning, "Sizing of Residential PV Battery Systems," Energy Procedia, vol. 46, pp. 78-87, 2014.

[19] A. Whiteman, J. Esparrago, S. Rueda, S. Elsayed, and I. Arkhipova, Renewable Energy Capacity Statistics 2019. Abu Dhabi: IRENA, 2019.

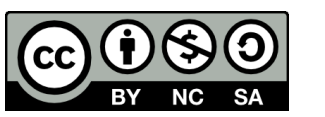
(C) 2021 by the authors. Submitted for possible open access publication under the terms and conditions of the Creative Commons Attribution CC BY-NC-SA 4.0 license (https://creativecommons.org/licenses/by-ncsa/4.0/deed.es). 